\title{
Red Lesion Detection Using Hough Transform and KNN Classifier for Diabetic Retinopathy Screening
}

\author{
Fayiza K T $\mathbf{T}^{1}$, Sherin Jabbar ${ }^{2}$ \\ ${ }^{1}$ PG Student, Computer Science and Engineering, MEA Engineering College, Kerala, India \\ ${ }^{2}$ Assistant Professor, Computer Science and Engineering, MEA Engineering College, Kerala, India
}

\begin{abstract}
Diabetic retinopathy is a major cause of blindness in the world. It will take more time to identify the clinical features such as microaneurysms, hemorrhages, exudates and cottonwool spots through manual inspection of fundus images. A computer assisted diagnosis system can help to reduce the burden on the ophthalmologist and rapidly identify the most severe cases. An efficient approach for red lesion detection in fundus image is proposed. The fundus image is preprocessed and Hough transform is used to identify the regions having red lesions. A KNN classifier is used to train the feature which classifies the images according to their probability of being normal or with disease.
\end{abstract}

Keywords: Diabetic retinopathy, Microaneurysm, Hemorrhages, Thresholding, Hough Transform, KNN classifier, Automatic detection

\section{Introduction}

Diabetic retinopathy (DR) is an important cause of blindness in the working population. This eye disorder is the most frequent microvascular complication of diabetes. Diabetes damages the macro and microvascular system. Usually the eye is one of the first places where this becomes apparent. When the microvascular system in the retina is progressively damaged, vision impairment and blindness can occur. Fig. 1 describes the difference between the vision of a normal person and a person with diabetic retinopathy.
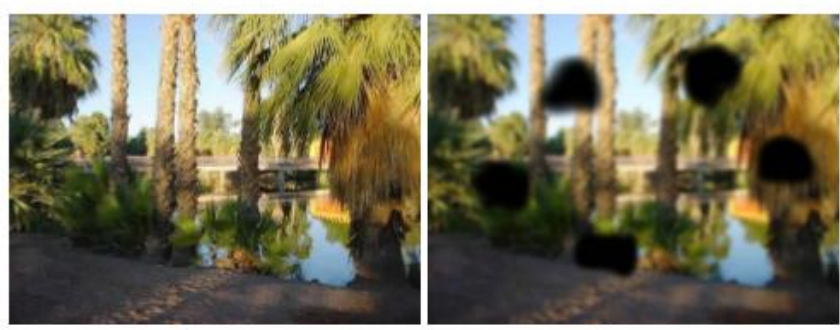

Figure 1: (a) Normal Vision (b) Vision with Advanced Diabetic Retinopathy

Even though diabetes itself cannot be cured at this time, DR can be successfully treated by laser surgery and strict glucose control. In order for the treatment to be effective, early detection is important. Most patients with diabetes will finally develop DR. The presence of diabetic retinopathy can be detected by examining the eye for its characteristic features. Fig. 2 shows the examples of retinal images containing symptoms of diabetic retinopathy, (a) microaneurysms, the contrast of the enlarged section has been enhanced, (b) hemorrhages (c) exudates (d) cottonwool spot.

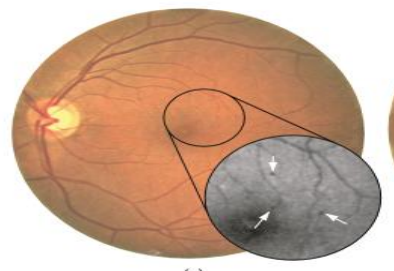

(a)
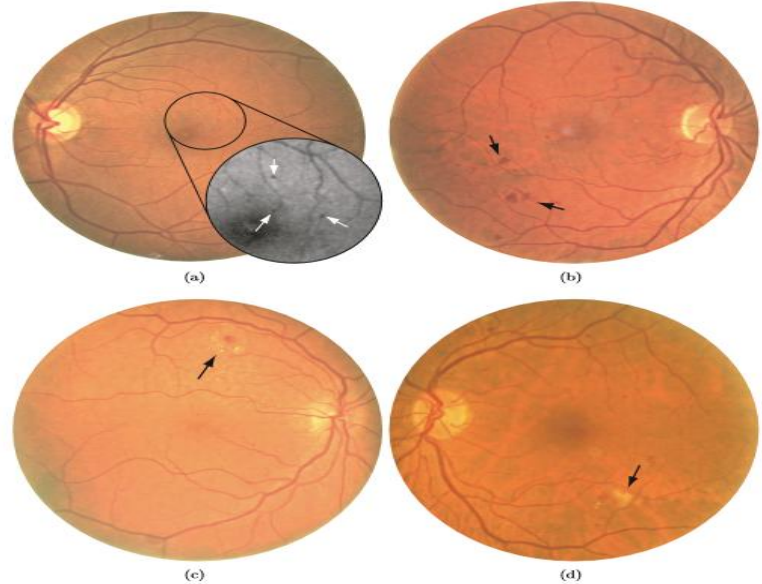

(b)

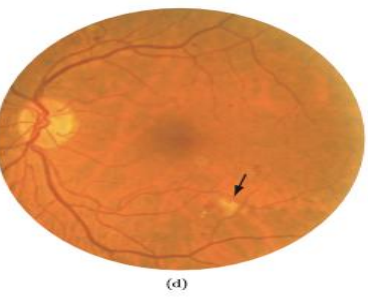

Figure 2: Examples of Retinal Images Containing Symptoms of Diabetic Retinopathy

Diabetic retinopathy can be classified as proliferative diabetic retinopathy and nonproliferative diabetic retinopathy [4]. NPDR occurs when the blood vessels inside the retina are damaged and leak extra fluid and small amounts of blood onto the retina. With this condition, retina becomes wet and swollen. Three subclasses of NPDR are

- Mild

- Moderate

- Severe

PDR is an advanced stage of diabetic retinopathy. Growth of new abnormal blood vessels in different regions of the retina may lead to total blindness [5]. Fig. 3 shows different stages of diabetic retinopathy based on increasing severity [6]. The research focuses on the analysis of different red lesion detection techniques used in available automatic telemedicine systems for computer aided screening and grading of DR. 


\section{International Journal of Science and Research (IJSR) \\ ISSN (Online): 2319-7064}

Index Copernicus Value (2015): 78.96 | Impact Factor (2015): 6.391

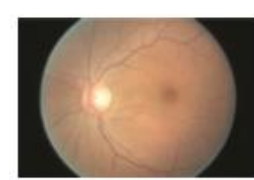

(a) No DR

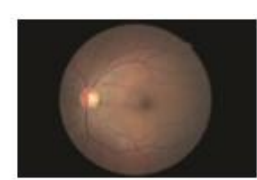

(b) Mild DR

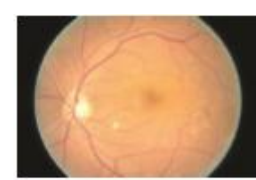

(c) Moderate DR

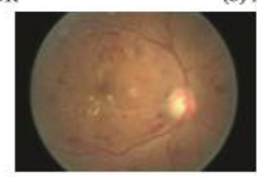

(d) Severe DR (e) Proliferative DR

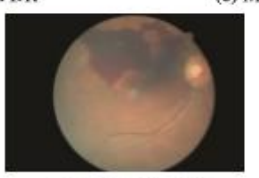

Figure 3: Stages of diabetic retinopathy [6]

\section{Literature Survey}

Diabetic retinopathy screening is a popular research area and a lot of researchers focus on and contribute towards the advancement of study in this area. Automatic detection techniques for DR screening were proposed in order to address the manual screening issues. The goal of automated approaches for screening is to identify the needs of referral for further treatment. Different techniques have been proposed and used for detecting the red lesions in order to produce an efficient and reliable detection system.

The process of analysing retinal images involves series of steps. Image acquisition, image preprocessing, candidate feature extraction and classification. All these steps includes various techniques or algorithms. Most of them uses a threshold based method to segment the image and blood vessels are removed. Intensity features are formulated to classify the images. Unfortunately, the main limitation to this approach is that most of the false positives at the vessel segmentation step are actually lesions. These lesions are removed along with the detected vessels and cannot retrieved in subsequent processing. Hough transform is used to find features of any shape in an image. Analytical curves such as lines, circles and ellipses can be detected using this method. Circular hough transform is used to find circles in imperfect image inputs. But its computational complexity and large memory requirement leads to slowness in performance. Dynamic shape features have proven to be robust features, highly capable of discriminating lesions from vessel segments. The concept of DSFs could be used in other applications having the objects to be detected do not show clear boundaries and are difficult to segment precisely [12].

\section{Proposed System}

In this paper, an automatic red lesion detection technique in retinal fundus image is presented. Fig. 4 shows the block diagram of the proposed system. The proposed method takes a color fundus image and the binary mask of its region of interest (ROI) as input. The region of interest is the circular area surrounded by a black background. The method comprises of six steps. First, spatial calibration is applied to adapt different image resolutions. Second, the input image is preprocessed to enhance the quality of the image via smoothing and normalization. Third, the optic disc is detected and eliminated. Fourth, candidate regions are identified. Fifth, the shape features are extracted for each candidate. Sixth, candidate lesions are classified according to their probability of being lesions and nonlesions. Each of these steps is described in the following subsections.

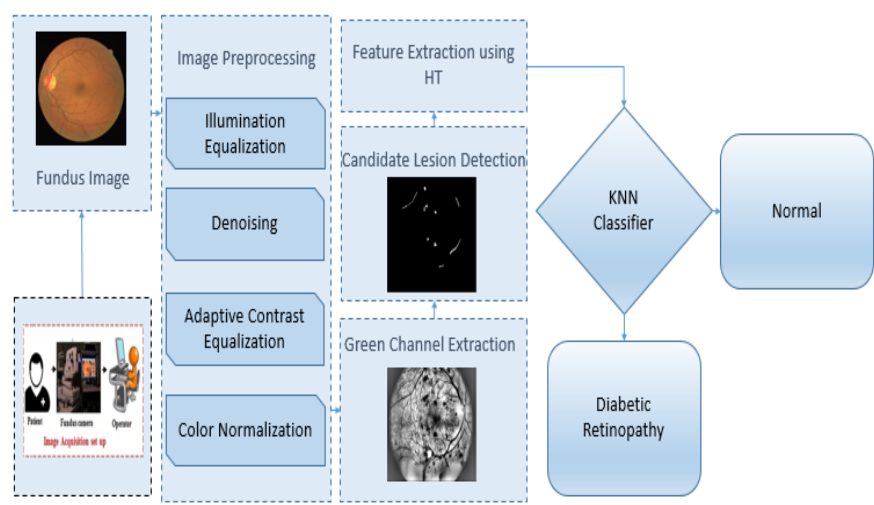

Figure 4: Block diagram

\subsection{Spatial calibration}

To support different image resolutions, a spatial calibration method introduced in [13] is used. Images are not resized, but the diameter D of the ROI is taken as a size invariant. It is used to set the kernel sizes of different filters. Three size parameters are used.

- $\mathrm{d} 1$ is the average radius of the OD

- $\mathrm{d} 2$ is the size of the smallest MA

- $\mathrm{d} 3$ is the size of the largest HE

These parameters are experimentally set to $\mathrm{d} 1=\mathrm{D} / 10$, $\mathrm{d} 2=\mathrm{D} / 360, \mathrm{~d} 3=\mathrm{D} / 28$.

\subsection{Image preprocessing}

Input image is preprocessed to improve contrast, remove noise and improve the quality of the image. Illumination equalization, denoising, adaptive contrast equalization and color normalization are the preprocessing techniques used in the proposed system.

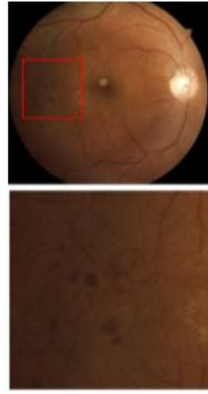

(a)

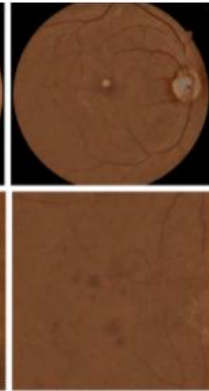

(b)

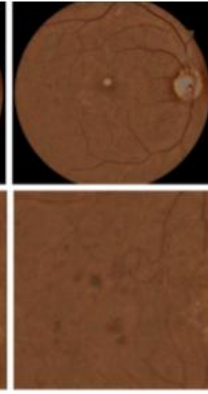

(c)

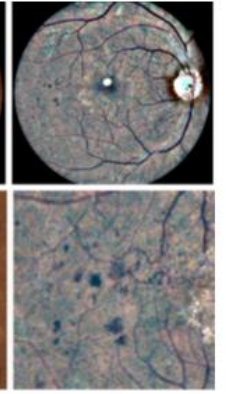

(d)
Figure 5: Preprocessing steps a) illumination equalization b) denoising c) adaptive contrast equalization d) color normalization [3]

\section{1) Illumination Equalization}

To remove uneven illumination of the image, illumination equalization method is used.

$$
I_{\text {ie }}=I+\mu-I * h_{M 1}
$$

The initial step is to estimate the background of the image. The image background is estimated by using a low pass filtering with a large kernel. The background estimated is 


\section{International Journal of Science and Research (IJSR) \\ ISSN (Online): 2319-7064}

Index Copernicus Value (2015): 78.96 | Impact Factor (2015): 6.391

then subtracted from the input image to correct potential shade variations. Finally an average intensity, $\mu$ of the original image is added in order to keep the same color range as in the original image.

\section{2) Denoising}

Denoising is the process of removing noise from images. It doesn't completely avoid the image noise, but certainly reduce them. Two basic approaches are

- Spatial filtering: Operate on a set of pixels related to a given pixel, usually by a sliding window. It is usually square but can be any shape.

- Transform domain filtering: It change the basis of signal space to aid some processing on the image data. Fourier transform and wavelet transform are some examples of these types.

A small mean filter of diameter $\mathrm{d} 2$ is applied to each color channel of the resulting image in order to remove noise from image.

\section{3) Adaptive contrast equalization}

To improve contrast, an adaptive contrast equalization method is used. First, the local standard deviation is computed for each pixel in a neighborhood of $\mathrm{d} 1$, for each color channel. It is then used to approximate the contrast drift. Area with low standard deviation indicate low contrast area which are enhanced using

$$
I_{c e}=I_{d n}+\frac{1}{I_{\text {std }}}\left(I_{d n} *\left(1-h_{M a}\right)\right)
$$

\section{4) Color normalization}

To obtain images with a standardized color range, histogram stretching and clipping in the range $\mu \pm 3 \sigma$ are performed. $\mu$ and $\sigma$ are the mean and standard deviation respectively.

\subsection{Optic disc removal}

The OD is a significant source of false positives and its removal is a necessary step in red lesion detection. After preprocessing location of the OD's center is estimated by using an entropy based approach. The OD is located in a high intensity region where the vessels have maximal directional entropy. A subsequent optimization step then estimates the OD's radius and refines its position.

\subsection{Candidate extraction}

Blood vessels and dark lesions have the highest contrast in the green channel [14]. The green channel is extracted from the preprocessed image and is denoted Gp. MAs and HEs appear as structures with local minimal intensity. Dynamics transformation [15] is used to rate regional minima according to their local contrast. Using this transformation, the minima is selected by thresholding the resulting contrast image. A selected minimum should have an intensity lower than the mean intensity in $\mathrm{Gp}$ to be considered a candidate region. This is supported by the fact that red lesions are darker than the retinal background.

\subsection{Hough Transform}

The hough is a feature extraction method which is used in image analysis, computer vision and digital image processing. It is used to find features of any shape in an image. By using a voting procedure, imperfect instances of objects within a certain class of shapes are identified. Regular shapes such as lines, circles and ellipses can be detected using this method. It is described as the transformation of a point in the $x-y$ plane to the parameter space. A parameter space is used in order to perform the voting procedure. Object candidates are obtained as local maxima. The circular hough transform has been previously proposed for the detection of microaneurysms in two types of photography modes.

- Retinal fluoresce in angiographic images

- Digital red free photographs

Here the application of hough transform is explored in another type of fundus photography modes, i.e. the color mode. Arbitrary shapes are detected by using Generalized hough transform. It is robust to partial or slightly deformed shapes and multiple occurrences of a shape during the same processing pass can be identified.

\subsection{Classification}

After extracting the candidate features the classification is performed which involves identifying the eye either as diseased or normal by discriminating between the vessels and actual lesions. The features selected are put in a KNN classifier for automatic classification.

\subsection{Implementation}

This section deals with details regarding the implementation of red lesion detection using hough transform and $\mathrm{KNN}$ classifier for diabetic retinopathy detection. The proposed methods are implemented using MATLAB2014. Two types of inputs are used.

1) Fundus Images with Signs of Diabetic Retinopathy (abnormal)

2) Normal Images

\section{Data Set}

Images in DIRETDB1 database is used for evaluation. The database consists of 89 color fundus images of which 84 contain at least mild non proliferative signs (MA) of the diabetic retinopathy and 5 are considered as normal which do not contain any signs of the diabetic retinopathy according to all experts participated in the evaluation. The images were taken in the Kuopio university hospital. The images were selected by the medical experts, but their distribution does not correspond to any typical population, i.e., the data is biased and no a priori information can be devised from it. The diabetic retinopathy abnormalities in the database are relatively small, but they appear near the macula which is considered to threaten the eyesight. Images were captured with the same 50 degree field of view digital fundus camera with varying imaging settings (flash intensity, shutter speed, aperture and gain) controlled by the system. 


\section{International Journal of Science and Research (IJSR)}

ISSN (Online): 2319-7064

Index Copernicus Value (2015): 78.96 | Impact Factor (2015): 6.391

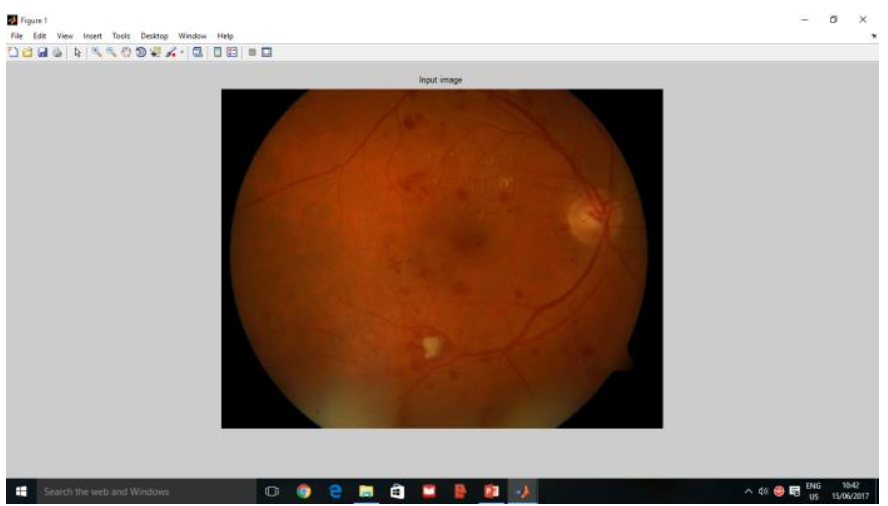

Figure 6: Input Image

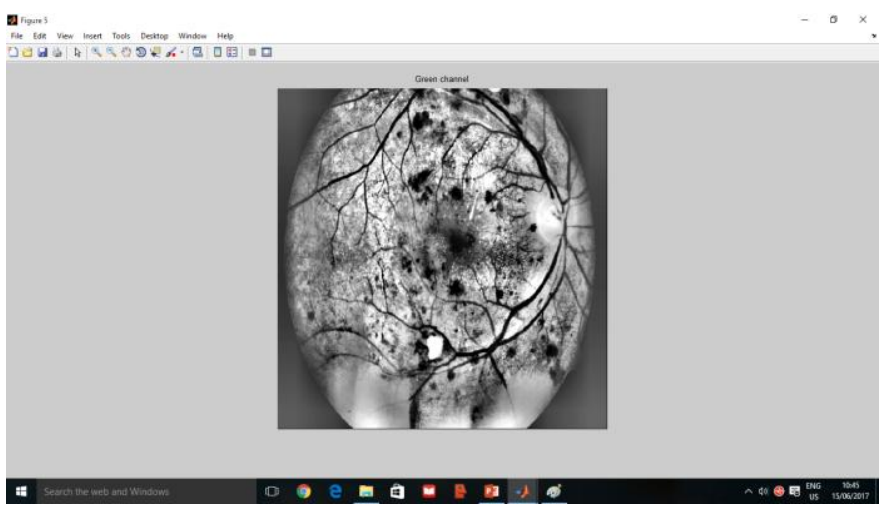

Figure 7: Result of Green Channel Extraction

After preprocessing the green channel of the image is extracted. Because blood vessels and red lesions have highest contrast in green channel. In the green channel, MAs and HEs appear as structures with local minimal intensity.

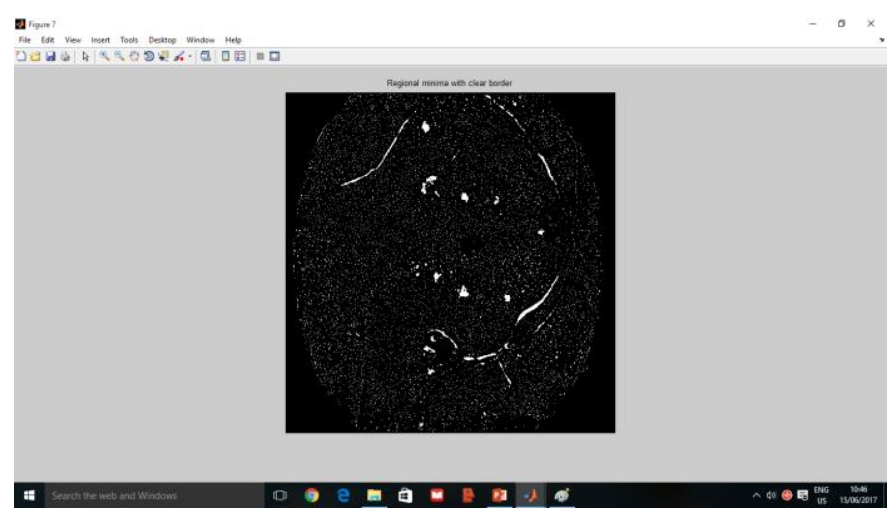

Figure 8: Result of Thresholding

Thresholding is applied to the resulting contrast image in order to select the minima (see Fig. 8). At this point, it is necessary to discard from the set of candidates as many local minima corresponding to noise as possible.

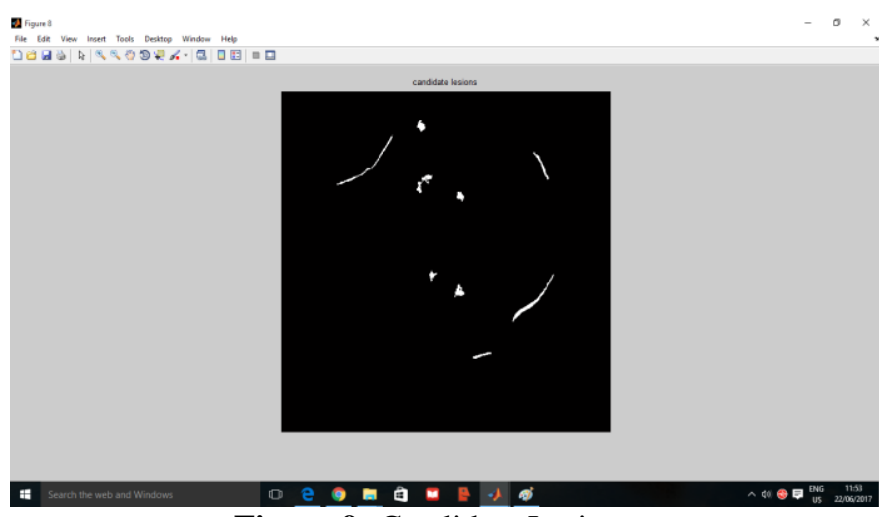

Figure 9: Candidate Lesions

In order to estimate the noise's intensity, the local standard deviation in a neighborhood of the size of the papilla is computed and consider the lowest standard deviation inside the ROI, which would correspond to a region in the retinal background with minimal signal intensity. Finally, a selected minimum should have an intensity lower than the mean intensity is considered as a candidate region (see Fig. 9). This is supported by the fact that red lesions are darker than the retinal background.

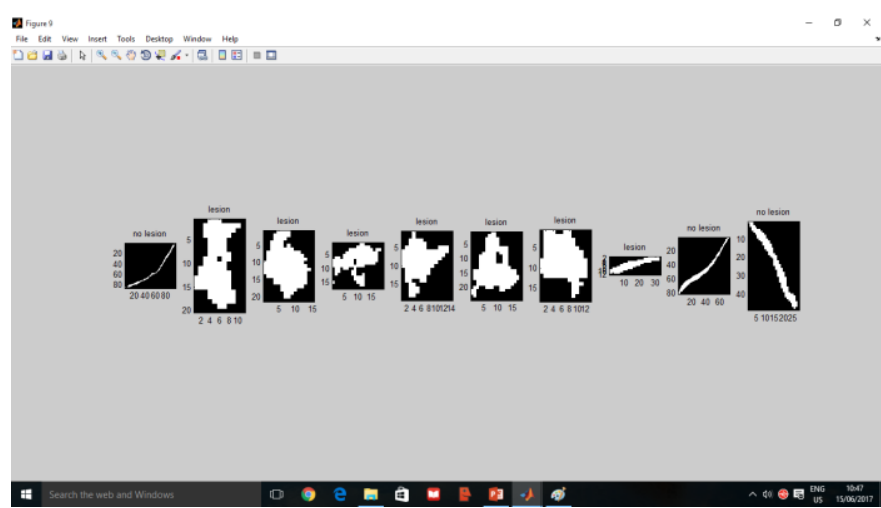

Figure 20: Feature Extracted Using Hough Transform

In proposed system, more features are selected to discriminate between vessels and lesions (see Fig.10). Most of the lesions are not detected when using DSFs [3]. This problem is solved in the proposed system. It is achieved by finding multiple occurrences of a shape (shape of lesions) during the same processing pass which is shown in Fig.11. The parameters of the shapes are stored in a look up table. A training model is created using these parameters and it is used to discriminate vessels from lesions. The parameters in the look up table of vessels are different from the parameters in the look up table of lesions. Finally a classifier is used to classify the new images according to their probability of being normal or with disease. 


\section{International Journal of Science and Research (IJSR) \\ ISSN (Online): 2319-7064}

Index Copernicus Value (2015): 78.96 | Impact Factor (2015): 6.391

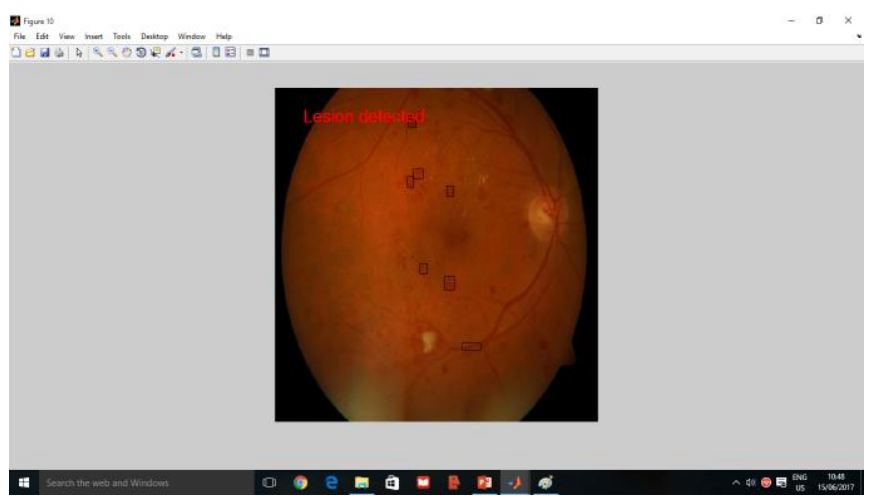

Figure 31: Final Result for Image with Signs of DR using HT

\section{Results}

A novel red lesion detection method based on hough transform was presented and evaluated on DIRETDB1 database. The result demonstrate the better performance of the proposed method in detecting red lesions in fundus images of different resolution and quality. 84 images from 89 images are correctly classified in proposed system whereas existing system classified 82 images. The implementation results (see Fig. 12) in turn prove the efficiency of the proposed method in terms of Accuracy.

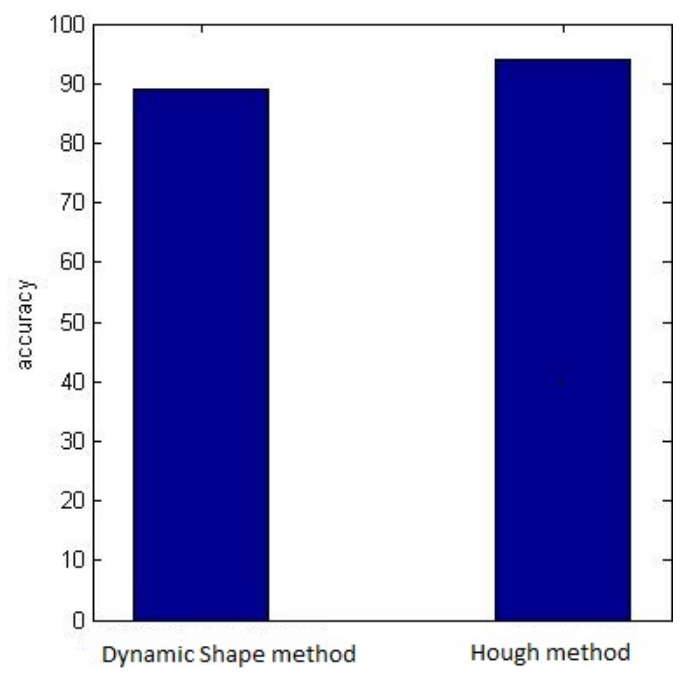

Figure 42: Implementation Results

\section{Conclusion and Future Work}

A novel red lesion detection method based on hough transform was presented and evaluated on different databases. The results demonstrate the better performance of the proposed method in detecting red lesions. This method was developed to detect red lesions from fundus images of different resolution, quality and taken from different acquisition systems and it is intended to help the ophthalmologists in the diabetic retinopathy screening process to detect symptoms faster and more easily. The proposed approach uses color fundus image as input, preprocessing is applied for removing the vignetting effect, noise removal and contrast enhancement. Then features of candidate lesions are extracted by using hough transform and selected features are given to train the classifier. The problem in separating the pathologies from small vessels are solved without removing the vessels. The retinal vessel which are discriminated from lesions could also be added in order to facilitate ophthalmologist decisions on laser treatment. Exudates and cottonwool spots detection could be added to the system in order to increase its ability to verify the degree of diabetic retinopathy. It will be useful to extend this work by developing a system to detect exudates and cottonwool spots.

\section{References}

[1] S. S. Rahim, C. Jayne, V. Palade and J. Shuttleworth, "Automatic detection of microaneurysms in colour fundus images for diabetic retinopathy screening," Neural Computing and Applications, pp. 1-16, 2015.

[2] M. U. Akram, S. Khalid, A. Tariq, S. A. Khan and F. Azam, "Detection and classification of retinal lesions for grading of diabetic retinopathy," Computers in biology and medicine, vol. 45, pp. 161-171, 2014.

[3] L. Seoud, T. Hurtut, J. Chelbi, F. Cheriet and J. P. Langlois, "Red lesion detection using dynamic shape features for diabetic retinopathy screening," IEEE transactions on medical imaging, vol. 35, no. 4, pp. 1116-1126, 2016.

[4] M. R. K. Mookiah, U. R. Acharya, C. K. Chua, C. M. Lim, E. Ng and A. Laude, "Computer aided diagnosis of diabetic retinopathy: A review, " Computers in biology and medicine, vol. 43, no. 12, pp. 2136-2155, 2013.

[5] O. Faust, R. Acharya, E. Y.-K. Ng, K.-H. Ng and J. S. Suri, "Algorithms for the automated detection of diabetic retinopathy using digital fundus images: a review," Journal of medical systems, vol. 36, no. 1, pp. 145-157, 2012.

[6] H. Pratt, F. Coenen, D. M. Broadbent, S. P. Harding, and Y. Zheng, "Convolutional neural networks for diabetic retinopathy," Procedia Computer Science, vol. 90, pp. 200-205, 2016.

[7] V. M. Mane, R. B. Kawadiwale and D. Jadhav, "Detection of red lesions in diabetic retinopathy affected fundus images," in Advance Computing Conference (IACC), 2015 IEEE International. IEEE, 2015, pp. 56-60.

[8] A. Sopharak, B. Uyyanonvara, S. Barman and T. H. Williamson, "Automatic detection of diabetic retinopathy exudates from nondilated retinal images using mathematical morphology methods," Computerized medical imaging and graphics, vol. 32, no. 8, pp. 720-727, 2008.

[9] S. A. Amiri, H. Hassanpour, M. Shahiri and R. Ghaderi, "Detection of microaneurysms in retinal angiography images using the circular hough transform," Journal of Advances in Computer Research, vol. 3, no. 1, pp. 1-12, 2012.

[10] S. A. A. Shah, A. Laude, I. Faye and T. B. Tang, "Automated microaneurysm detection in diabetic retinopathy using curvelet transform," Journal of biomedical optics, vol. 21, no. 10, pp. 101 404-101 404, 2016. 
[11]I. Soares, M. Castelo-Branco, and A. M. Pinheiro, "Microaneurysms detection using a novel neighborhood analysis," 2014.

[12] https://www.ijsr.net/conf/RCIP2017/RCIP20177.pdf

[13]X. Zhang et al., "Exudate detection in color retinal images for mass screening of diabetic retinopathy," Med. Image Anal., vol. 18, no. 7, pp. 1026-1043, 2014.

[14] T. Walter et al., "Automatic detection of microaneurysms in color fundusimages,"Med.ImageAnal., vol.11,no.6,pp.55566,2007.

[15]M. Grimaud, "A new measure of contrast: The dynamics," in SPIE Image Algebra Morphological Image Process., 1992, vol. 1769, pp. 292-305.

\section{Author Profile}

Fayiza $\mathbf{K} \mathbf{T}$ received her B. Tech degree in computer science and engineering from the MES College of Engineering, Kuttippurm, Kerala, in 2014. Right now she is pursuing her $\mathrm{M}$. Tech degree in computer science and engineering at MEA Engineering College, Kerala from 2015 to 2017 . Her research interests lies in Image Processing.

Sherin Jabbar is a post graduate in Computer Science and Engineering from MES College of Engineering, Kuttippuram. She graduated from Govt. Engineering College, Sreekrishnapuram, Palakkad. She is currently working as Assistant Professor in MEA Engineering

College, Kerala. Her research interest lies in Image Processing. 